Communications in Physics, Vol.21, No. 2 (2011), pp. 179-185

\title{
SYNTHESIS AND OPTICAL CHARACTERIZATION OF DYE DOPED IN ORMOSIL NANOSPHERES FOR BIOAPPLICATIONS
}

\author{
VU THI THUY DUONG, DO QUANG HOA, NGUYEN THI VAN, \\ NGHIEM THI HA LIEN, AND TRAN HONG NHUNG \\ Institute of Physics, VAST \\ J.C. BROCHON \\ Laboratoire de Biotechnologie et Pharmacologie Génétique Appliquée, \\ ENS Cachan, France.
}

\begin{abstract}
Dye-doped ORMOSIL (organically modified silicate) nanoparticles (NPs) have significant advantages over single-dye labeling in signal amplification, photostability and surface modification for various biological applications. The dyes: Rhodamine $6 G(R 6 G)$ and Rhodamine $B(R B)$ were successfully incorporated into ORMOSIL nanoparticles fabricated by micellar nanochemistry from Trimethoxysilane $\mathrm{CH}_{3} \mathrm{Si}\left(\mathrm{OCH}_{3}\right)_{3}$ precursor. The optical characterization of dye-doped ORMOSIL NPs was studied in comparison with its of free dye in solution. The results shown that the photostability of ORMOSIL dye doped nanospheres is much improved in comparison with its of dye in solution. Other studies of the photophysical properties such as anisotropy, fluorescence lifetime and energy transfer were also done.
\end{abstract}

\section{INTRODUCTION}

Dyes doped in silica and in polymer-based nanoparticle (PNs) have been investigated in the last decade [1-6]. Weihong Tan and co-workers showed that silica based NPs are less aggregate and little dye leakage in comparison with polymer based NPs. The size of silica particles remains unchanged while changing solvent polarity [2]. In silica-based NPs dye molecules are incorporated into the silica network, in turn silica host. Silica NPs possess three important properties that have made them highly useful in bioanalysis: highly optical intensity, excellent photostability, and easy surface modification with various functional groups (e.g., amine, thiol, carboxyl). Furthermore, silica is used because it is chemically inert; it protects the embedded dyes of the particle from the outside environments. Silica matrix is optically transparent that allows excitation and emission light to pass through the silica matrix. Dye can be attached to the NPs surface or can be embedded inside the particles by noncovalent or covalent bonds. These NPs can be also modified with biomolecules for biological applications. Silica NPs applications have been using for detection the immunogen, for cellular imaging, multiplexed bioanalysis, and nucleic acid detection [3]. These are two main methods used in the synthesis silica based fluorescent NPs: the Stober method and the reverse-micelle method or water in oil micro emulsion system. NPs formed via Stober method are typically large and non uniform but this method is simple to realize and can be carried out in short time and the solvents used in this method are non toxic: water or ethanol. Stober method has been modified to easily 
incorporate organic dye molecules inside the silica NPs and reform high uniform beads. NPs produced by using the water in oil system usually give a high degree of uniformity and are well dispersed in water but this method is only use to incorporate inorganic dyes [3]. This work presents the results in synthesis and optical properties study the dye-doped ORMOSIL nanoparticles (DDONPs) by modified Stöber method from Trimethoxysilane $\mathrm{CH}_{3} \mathrm{Si}\left(\mathrm{OCH}_{3}\right)_{3}$ precursor.

\section{MATERIALS AND EXPERIMENT}

\section{Reagents}

Methyltrimethoxysilane (MTMOS), Aminopropyl triethoxysilane (APTEOS), dimethylsulfoxide (DMSO), Clorotrimethylsilane, aqueous Ammonia solution 25\%, cosurfactant butanol-1 were purchased from Merck. Rhodamine 6G (R6G) and Rhodamine B (RB) dyes were from Exiton. Surfactant Aerosol-OT (AOT) (96\%) was purchased from Fluka. Dialysis tubing MWCO=10000 was purchased from Sigma-Aldrich.

\section{Synthesis}

Modified Stöber method was used for the synthesis ORMOSIL particles. A cosurfactant mixture of AOT and butanol1 with ratio AOT: butanol 1 (g): 1.82 (ml) was prepared using supersonic till optically clear. In order to adjust the sizes of particles, the quantities of this mixed co-surfactant have been changed [4]. Then in the $200 \mu \mathrm{l}$ MTMOS precursor was added $1.22 \mathrm{ml}$ above co-surfactant. This mixture was diluted with $20 \mathrm{ml}$ double distilled water and was added $100 \mu \mathrm{l}$ dye in DMSO while stirring which give a micellar system MTMOS + DMSO (dye) /AOT (butanol-1) / water. For void NPs, 100 $\mu \mathrm{l}$ DMSO without dye was added. The dye doped ORMOSIL NPs with an amino group binding on the surface start to form when $40 \mu \mathrm{l}$ APTEOS or aqueous Ammonia was added to the micelle system. For NPs without amino group on the surface, other catalyst was replacing APTEOS. After $1 \mathrm{~h}$ of reaction, a $20 \mu \mathrm{l}$ clorotrimethylsilane was added to quench the remaining silanol groups in the surface of NPs. Next, this system was stirring for $20 \mathrm{~h}$ at room temperature. The obtained solution then was dialyzed in a 10000 MWCO dialysis tubing against water for a week in order to remove the remaining chemical agents and all the surfactant AOT and butanol-1.

\section{Energy transfer}

The RB and R6G dyes were added into the NPs with different concentration combinations as reported in table 2 . Some samples were doped with only one type of dye at different concentrations. In the case of R6G, it is noted as samples \#1, with dye concentration respectively of $1 \times 10^{-2} \mathrm{~mol} / \mathrm{l}$, and for $\mathrm{RB}$ as samples \#5, \#6 and \#7, with dye concentration respectively of $0.5 \times 10^{-2}, 0.25 \times 10^{-2}$ and $0.1 \times 10^{-2} \mathrm{~mol} / \mathrm{l}$. Other samples were co-doped with both dyes, keeping constant the R6G concentration equal to $1 \times 10^{-2}$ $\mathrm{mol} / \mathrm{l}$ and changing RB concentration as $0.5 \times 10^{-2}, 0.25 \times 10^{-2}$ and $0.1 \times 10^{-2} \mathrm{~mol} / \mathrm{l}$ (samples \#3 to \#5). 


\section{Equipments}

High-resolution scanning electron microscopy (HSEM) Hitachi-S480 was used to determine the shape and size of particles. The chemical structure of NPs was studied using a Micro-Raman spectrograph LAMBRAM-1 with a laser He-Ne $632.8 \mathrm{~nm}$ as excitation source at room temperature and Impact 410 Nicolet FTIR spectrophotometer. Absorption spectra were measured using JASCO-V570-UV-VIS-NIR spectrometer. The fluorescence lifetime was detected using a Nd: $\mathrm{YVO}_{4}$ subpicosecond home-made laser pumped by diode laser with a repetition rate reduced to $8 \mathrm{MHz}$. A fast 300 ps risetime Hamamatsu silicon photodiode and Tektronix oscilloscope TDS $72541.5 \mathrm{GHz}$ were used as detection system. The fluorescence and anisotropy spectra were measured using laser Cd-He $442 \mathrm{~nm}$ as light source and a Spectrometer (MicroSpec 2300i Acton Research Corp.) with a cooled CCD camera (Pixis 256 Princeton Instruments) as the detection system. Polarization was acquired with excitation and emission polarisers in Vertical-Vertical (VV) and VerticalHorizontal (VH) configurations. The instrumental correction G-factor was determined with the same configuration. The emission polarization is given by:

$$
p=\frac{I_{V V}-G \cdot I_{V H}}{I_{V V}+G \cdot I_{V H}} \text { or anisotropy } r=\frac{I_{V V}-G \cdot I_{V H}}{I_{V V}+2 G \cdot I_{V H}}, \text { where } G=\frac{I_{V V}^{\prime}}{I_{V H}^{\prime}}
$$

$I_{V V}$ and $I_{V H}$ are the fluorescence intensity of sample at Vertical-Vertical and VerticalHorizontal positions, respectively. $I_{V V}$ and $I_{V H}$ are the fluorescence intensity of nonpolarized sample at the sample configuration. The fluorescence intensity of NPs vs. time was investigated using laser He-Ne $543 \mathrm{~nm}$ as an excitation source and a Hamamatsu $\mathrm{Si}$ photodiode for the detection.

\section{RESULTS AND DISCUSSIONS}

\section{Size, shape and chemical structure}

The HSEM image of dye doped NPs is present in Fig. 1a). It shows that the particle shape is spherical with the average diameter of $60-80 \mathrm{~nm}$ with high monodispersion.

The chemical structure of NPs are determined by analyzing the micro Raman and FTIR spectra of both void ormosil NPs made from MTMOS precursor with and without APTEOS catalyze. As it is shown in Figure 1b) and c), the Raman and FTIR spectra of NPs made without APTEOS are composed from two principal groups: the vibration bands of $\mathrm{SiO}_{2}$ network and its of methyl group bound to silicon atom $\left(\mathrm{Si}-\mathrm{CH}_{3}\right)$. The Raman and FTIR spectra of NPs made with APTEOS are mainly similar to those of non-APTEOS but the bands of vibration of amino groups $\mathrm{NH}_{2}$. So, it is clear that the use of APTEOS as catalyze gives us the NPs with amino groups bound to silicon atom on the surface. This amino group will play biocompatible role in the bioapplications.

\section{Fluorescence spectra and Anisotropy}

In order to compare the optical properties of the dye doped in NPs and bare dye, the R6G and RB dyes were diluted in water with $0.2 \% \mathrm{DMSO}(\mathrm{v} / \mathrm{v})$ that its intensity of absorption is the same as in NPs. The absorption and fluorescence spectra of RB and R6G in water and in NPs are depicted in Fig. 2. As it is shown in Fig. 2, the absorption and 


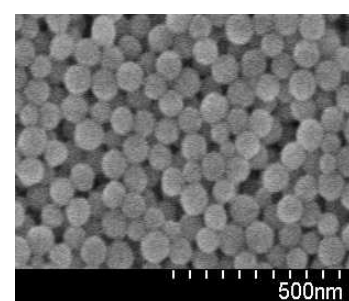

(a)

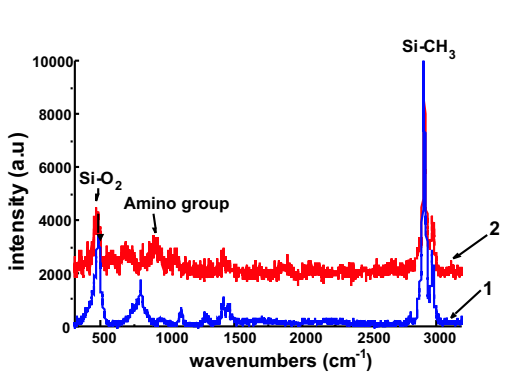

(b)

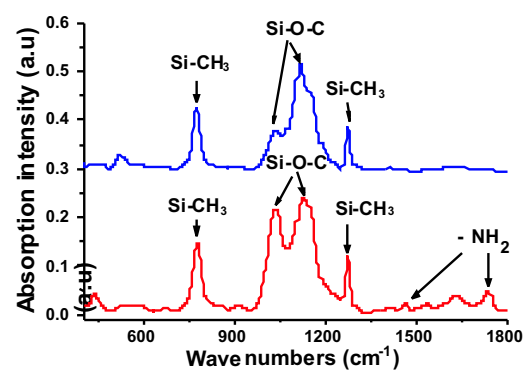

(c)

Fig. 1. (a) HSEM image of NPs; (b) Raman spectra of void NPs; curve 1: spectrum of NPs made without APTEOS, curve 2: spectrum of NPs made with ATEOS; (c) FTIR spectra of NPs; upper curve: spectrum of NPs made without APTEOS, lower curve: spectrum of NPs made with ATEOS.

fluorescence spectra of R6G and RB in water and in NPs are similar but a little red-shift of the spectral maxima of dyes in NPs in comparison with its of dyes in water.

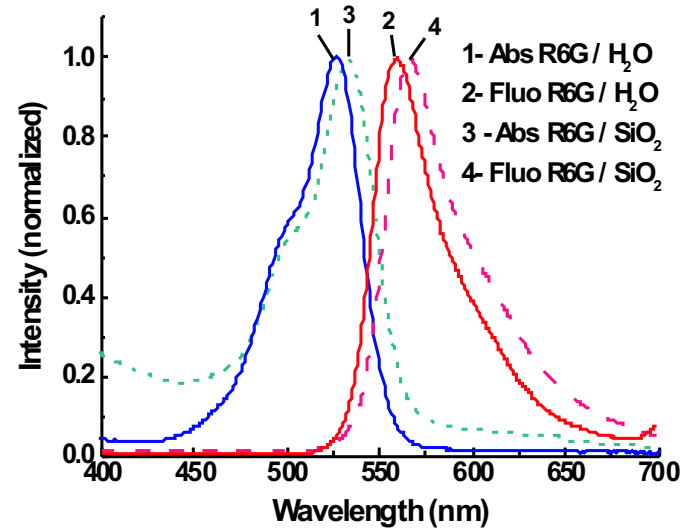

(a)

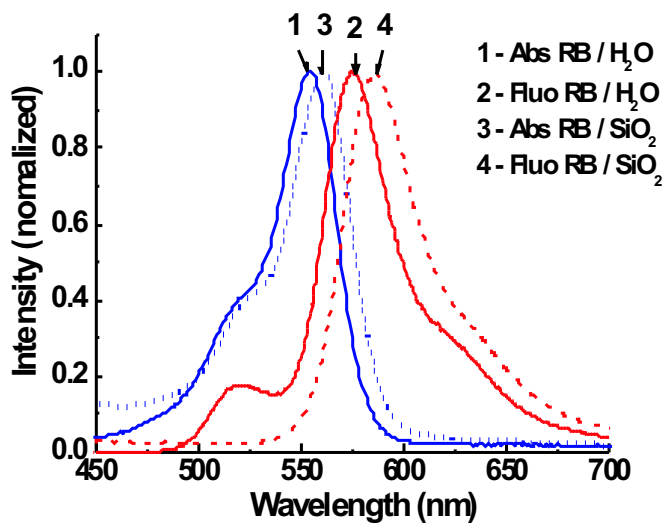

(b)

Fig. 2. Absorption and fluorescence spectra of R6G (a) and RB (b).

The fluorescence lifetime measurements recorded in Table 1 shows that the lifetimes lightly change for dyes in NPs All these facts indicate the perturbations of dyes in NPs; dyes interact with NPss matrix and between themselves.

For the R6G and RB dyes in water, the emission polarization was not obtained. For the R6G dye in NPs which the particles diameter is about $30 \mathrm{~nm}$, the polarization is about $0.18 \pm 0.018$. For the RB dye in NPs which the particles diameter is about $70 \mathrm{~nm}$, the polarization is $0.07 \pm 0.007$. Anisotropy of dyes doped in NPs emission is attributable to dye-matrix interactions. Here, it seems to be that particle size might indeed be a 
Table 1. Spectral data of dyes in water and in NPs: absorption maximum wavelength $\lambda_{A}$, fluorescence maximum wavelength $\lambda_{F}$, its full width at half maximum (FWHM) and lifetime.

\begin{tabular}{ccccccc}
\hline \hline \multirow{2}{*}{ Dye } & \multicolumn{2}{c}{ Absorption } & \multicolumn{2}{c}{ Emission } & Fluorescence \\
& & $\lambda_{A}$ & FWHM & $\lambda_{F}$ & FWHM & Lifetime \\
$(\mathrm{nm})$ & $(\mathrm{nm})$ & $(\mathrm{nm})$ & $(\mathrm{nm})$ & $(\mathrm{ns})$ \\
\hline \hline \multirow{2}{*}{ R6G } & Water & 526 & 50 & 554 & 44 & 5.2 \\
& NPs & 533 & 54 & 560 & 51 & 4.2 \\
\multirow{2}{*}{ RB } & Water & 559 & 36 & 576 & 40 & 1.4 \\
& NPs & 565 & 38 & 586 & 46 & 1.8 \\
\hline \hline
\end{tabular}

major factor on emission anisotropy. Other investigation must to be done in order to clear whether the influence of size operates primarily at the level of emission and / or excitation.

\section{Photostability}

Fig. 3 shows the fluorescence intensity vs. time curves of R6G and Rhodamine B dyes in water and in NPs upon a He-Ne laser irradiation at $543 \mathrm{~nm}$ and $3.2 \mathrm{~mW} / \mathrm{cm}^{2}$. The fluorescence intensity of dyes in water was down to the half after about 90 minutes of irradiation while its of dyes in NPs remains unchanging after 140 minutes of lighting up.

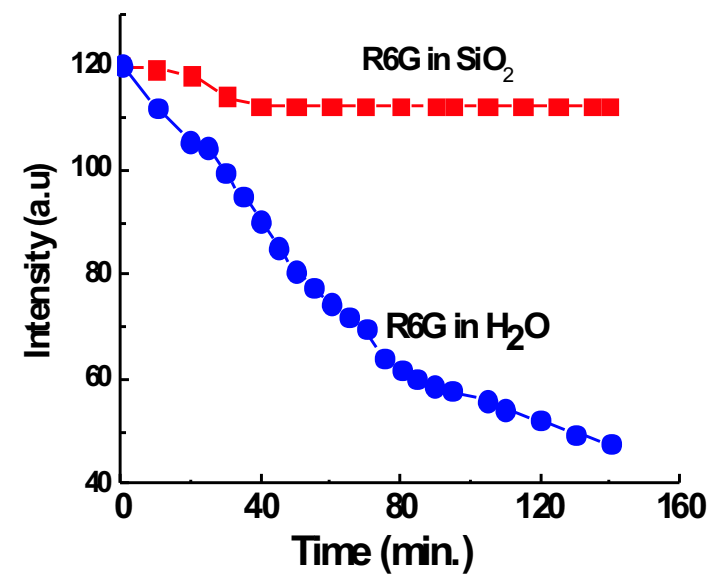

(a)

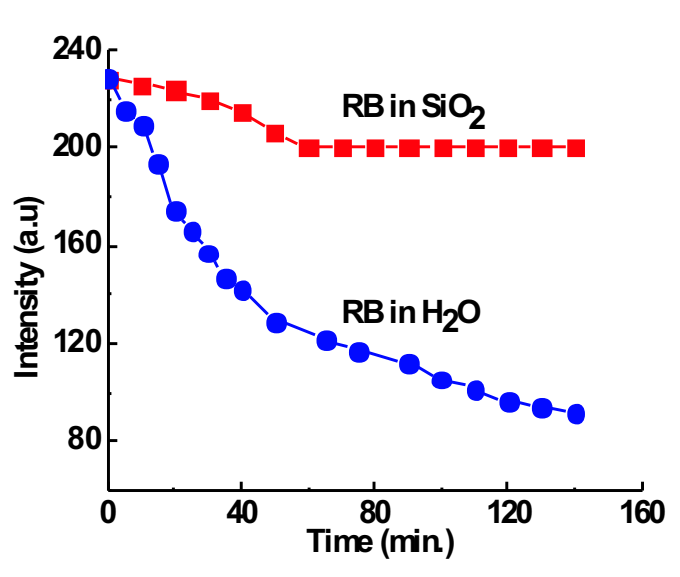

(b)

Fig. 3. Emission Intensity of R6G (a) and RB (b) in water and in NPs vs. time. Excitation by laser He-Ne at $543 \mathrm{~nm}, 3.2 \mathrm{~mW} / \mathrm{cm}^{2}$.

\section{Energy transfer}

The results of energy transfer from R6G to RB dye molecules in NPs studies are presented in Fig. 4 and Table 2. From Fig. 4, it is clear that the fluorescence intensity RB dye molecules in samples with dye mixture (\# 2- \#4) is higher than its in samples with 
single RB dye (\#5 - \#7), due to energy transfer from R6G to RB dye molecules. The energy transfer parameters such as radiative transfer efficiency $\eta_{R}$ and transfer probability $P_{D A}$ are calculated and presented in Table 2 . The half quenching concentration and critical radius were estimated; $[A]_{1 / 2}=0.1 \times 10^{-2}(\mathrm{~mol} / \mathrm{l}), R_{0}=73.5 \AA$, respectively. The details of energy transfer studies must be presented in an another publication.

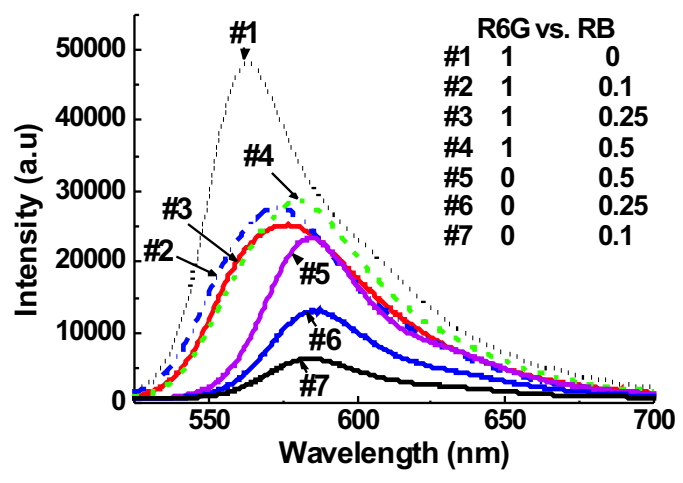

Fig. 4. Fluorescence spectra of R6G, RB and dye mixture samples: R6G (\#1), $\mathrm{RB}(\# 5-\# 7)$ and dye mixture $(\# 2 \quad \# 4)$.

Table 2. Spectral and energy transfer data of R6G and RB: $\lambda_{F}$, transfer efficiency $\eta_{R}$ and transfer probability $P_{D A}$

\begin{tabular}{cccccc}
\hline \hline No & \multicolumn{2}{c}{ Concentration } \\
$\left(\times 10^{-2}\right.$ & $\mathrm{mol} / \mathrm{l})$ & $\begin{array}{c}\lambda_{F} \\
(\mathrm{~nm})\end{array}$ & $\begin{array}{c}\eta_{R} \\
(\%)\end{array}$ & $\begin{array}{c}\text { PDA } \\
\left(\times 10^{6} \mathrm{~s}^{-1}\right)\end{array}$ \\
\hline \hline 1 & 1 & 0 & 566 & 0 & 0 \\
2 & 1 & 0.1 & 575 & 61.3 & 365.2 \\
3 & 1 & 0.25 & 577 & 75.9 & 725.7 \\
4 & 1 & 0.5 & 580 & 80.8 & 967.8 \\
5 & 0 & 0.5 & 584 & 0 & 0 \\
6 & 0 & 0.25 & 584 & 0 & 0 \\
7 & 0 & 0.1 & 585 & 0 & 0 \\
\hline \hline
\end{tabular}

\section{CONCLUSION}

The aqueous R6G and RB doped ORMOSIL NPs have been synthesized by modified Stöber method. The NPs are uniform spheres of diameter from $30 \mathrm{~nm}$ to $100 \mathrm{~nm}$, monodispersed, with the amino NH2 group on the surface. The photostability of R6G and $\mathrm{RB}$ dyes doped inside ORMOSIL NPs are much higher than those of dyes in water. The fluorescence polarization was observed for doped in NPs dyes, which is attributable to dye-matrix interactions. The effect of energy transfer from R6G to RB dyes have been 
observed for the R6B \& RB dye mixtures doped in ORMOSIL NPs, that its fluorescence spectra can be filled from 566 to $584 \mathrm{~nm}$ and emission efficiencies are higher than these of single dyes. Other studies of the energy transfer between dyes in NPs and the application of particles for cell labeling must be done.

\section{ACKNOWLEDGMENTS}

The work was partly supported by National Foundation for Science and Technology Development, Project No. 103.06101.09.

\section{REFERENCES}

[1] Jilin Yan, M. Carmen Rstevez, JoshuaE. Smith, Kemin Wang, Xiaoxiao He, Lin Wang, and Weihong Tan, Nanotoday 2 (3) (2007) 44-50.

[2] W. Tan, et al., Med.Res. Rev 24 (2004) 621.

[3] Joshua E. Smith, Lin Wang, Weihong Tan, Anal. Chem. 25 (9) (2006) 848-855.

[4] Indrajit Roy, Tymish Y. Ohulchanskyy, Haridas E. Pudavar, Earl J. Bergey, Alain R. Oseroff, J. Morgan, Thomas J. Dougherty, Paras N. Prasad, PNAS 125 (2007), 279-284.

[5] Nathaiel L. Rosi and Chad A. Mirkin, Chem. Rev. 105 (2005), 1547-1562.

[6] A. Van Blaaderen, and A. Vrij, J. Colloid and Iterface Science 156 (1993), 1-18.

Received 29 October 2010. 